\title{
Effects of Nose Bluntness on Stability of Hypersonic Boundary Layers over Blunt Cone
}

\author{
K. Kara* \\ Department of Aerospace Engineering \\ Old Dominion University, Norfolk, VA, 23529 \\ P. Balakumar ${ }^{\dagger}$ \\ Flow Physics and Control Branch \\ NASA Langley Research Center, Hampton, VA 23581 \\ O. A. Kandil ${ }^{\star}$ \\ Department of Aerospace Engineering \\ Old Dominion University, Norfolk, VA, 23529
}

\begin{abstract}
Receptivity and stability of hypersonic boundary layers are numerically investigated for boundary layer flows over a 5-degree straight cone at a free-stream Mach number of 6.0. To compute the shock and the interaction of shock with the instability waves, we solve the NavierStokes equations in axisymmetric coordinates. The governing equations are solved using the $5^{\text {th }}-$ order accurate weighted essentially non-oscillatory (WENO) scheme for space discretization and using third-order total-variation-diminishing (TVD) Runge-Kutta scheme for time integration. After the mean flow field is computed, disturbances are introduced at the upstream end of the computational domain. Generation of instability waves from leading edge region and receptivity of boundary layer to slow acoustic waves are investigated. Computations are performed for a cone with nose radii of $0.001,0.05$ and 0.10 inches that give Reynolds numbers based on the nose radii ranging from 650 to 130,000 . The linear stability results showed that the bluntness has a strong stabilizing effect on the stability of axisymmetric boundary layers. The transition Reynolds number for a cone with the nose Reynolds number of 65,000 is increased by a factor of 1.82 compared to that for a sharp cone. The receptivity coefficient for a sharp cone is about 4.23 and it is very small, $\sim 10^{-3}$, for large bluntness.
\end{abstract}

\section{Introduction}

$\mathrm{T}$ ransition from laminar to turbulent state in shear flows occurs due to evolution and interaction of different disturbances inside the shear layer. Though there are several mechanisms and routes to go from a laminar to a turbulent state, most of them generally follow these fundamental processes; a) receptivity b) linear instability c) nonlinear instability and saturation d) secondary instability and breakdown to turbulence.

The transition onset mainly depends on the boundary layer characteristics and on the frequency, wave number distributions, and the amplitudes of the disturbances that enter the boundary layer. The boundary layer profiles depend on the flow parameters such as Mach number, Reynolds number, wall temperature, and model geometry. In supersonic and hypersonic boundary layers one important geometrical parameter is the nose bluntness. The effects of bluntness on transition have been studied experimentally and numerically by many researchers ${ }^{1-6}$. It was found that the bluntness generally stabilizes the boundary layer. The critical Reynolds numbers for blunt cones are much higher compared to that for sharp cones. However, the transition Reynolds number increased only by a factor of two compared to the sharp cones. It was identified that the entropy layer that is formed near the bow shock region persists for a long distance downstream and makes the boundary layer more stable compared to the sharp cone case.

${ }^{*}$ Graduate Research Assistant, Student Member AIAA

${ }^{\dagger}$ Research Scientist, Member AIAA

* Professor \& Eminent Scholar, Associate Fellow AIAA 
After the entropy layer and the boundary layer that is developing along the surface merges together, the boundary layer becomes unstable. It was also found that in addition to first and the second modes instability waves, other inviscid type disturbances grow inside the entropy layer. It was also observed that with increasing bluntness the stabilizing trend is reversed in axisymmetric boundary layers. Another influence of the bluntness is in the generation of instability waves near the leading edge region. The objectives of this work are to estimate the stabilizing effect of the bluntness on the hypersonic boundary layers over blunt cones, to estimate the transition Reynolds number based on the $\mathrm{e}^{\mathrm{N}}$ criteria, and to compute the receptivity coefficient of the instability waves generated inside the boundary layer. To investigate the effect of the Reynolds number based on the nose bluntness, simulations are performed at different leading edge radii $r_{0}=0.001,0.05$ and 0.10 inches at a unit Reynolds number of $7.8 * 10^{6} / \mathrm{ft}$ for a 5 -deg. half-angle cone. To differentiate the unit Reynolds number effect from the nose Reynolds number effect, one simulation is performed at a higher unit Reynolds number of $15.6^{*} 10^{6} / \mathrm{ft}$ with 0.05 inches bluntness. These parameters yield the Reynolds number based on the nose radius to vary from 650 to 130,000 . The results consist of: (1) mean flow profiles, linear stability and transition onset Reynolds numbers at different bluntness, and (2) receptivity coefficients for different bluntness. A schematic diagram of the computational set up is depicted in Fig. 1.

\section{Literature Review}

Stetson ${ }^{7}$ carried out boundary layer experiments to investigate the effects of nose tip bluntness on 8-degree half angle cone containing two rays of thermocouples in AEDC Tunnel $\mathrm{F}$ at Mach 6. The location of boundary layer transition was obtained from heat transfer measurements. It was found that the small nose tip bluntness had a stabilizing effect upon boundary layer when transition occurred at locations where the entropy layer nearly swallowed. Also Stetson et al. ${ }^{1}$ investigated the stability of the laminar boundary layer experimentally on a blunt, 7 degree half angle cone at Mach 8 and identified disturbances growing outside the boundary layer, in the entropy layer, indicating the existence of an inviscid instability. Recently Maslov et al. ${ }^{8,9}$ conducted stability experiments on sharp and blunt cones at Mach 5.92. Rufer and Schneider ${ }^{10}$ measured mass flux profiles over 7-degree half angle sharp and blunt ( $0.020 \mathrm{in}$. radius) cones to study the amplitude and growth of instability waves. Also Schneider ${ }^{11,12}$ published additional stability experimental results of Stetson's ${ }^{1}$ experiment. An experimental investigation was conducted on a 5-degree half-angle cone in a conventional Mach 6 wind tunnel done by Horvath et $\mathrm{al}^{13}$ to examine the effects of facility noise on boundary layer transition. In addition, the model nose tip radius was varied from 0.0001 in. to 0.0625 in. to examine the effect of bluntness on transition onset.

Malik et al. ${ }^{3}$ computed the effect of nose bluntness on boundary layer instability for Mach 8 flow past a 7degree half angle cone. They included the entropy-layer effect using Parabolized Navier-Stokes equations. It is concluded that nose bluntness stabilizes the boundary layer and the effect of unit Reynolds number in the aeroballistic range data of Potter ${ }^{14}$ was a nose bluntness effect. Rosenboom et al ${ }^{15}$ and Zhong ${ }^{16}$ did further study on the effect of nose bluntness on the linear stability of hypersonic flow over Stetson's blunt cone and focused on the transition reversal phenomenon. However, no instability reversal observed as the nose radius increased in both studies. Their results indicated that to understand the cause of the transition reversal phenomenon it is necessary to conduct further studies on the nose bluntness. Balakumar ${ }^{17}$ performed computations for a blunt flat plate with thicknesses from 0.0001 to 0.01 inches and a wedge of 10-degree half angle with different leading edge radii 0.001 and 0.01 inches to find out the effect of nose bluntness on the stability of two dimensional boundary layers. He found that the bluntness has a strong stabilizing effect on the stability of two dimensional boundary layers and the boundary layers on wedges are far more stable that on blunt flat plates.

Here, we employ a fifth order weighted essentially non-oscillatory (WENO) scheme for spatial discretization and use third order total variation diminishing (TVD) Runge-Kutta scheme for time integration to solve for the hypersonic boundary layer receptivity problem. For this study, we have selected the flow conditions and geometry used by Horvath et $\mathrm{al}^{13}$. The nose radii are varied from $r_{0}=0.001$ to 0.10 inches and the flow around the leading edge is resolved by using a sufficiently dense grid. We assume adiabatic wall conditions for steady flow computations. 


\section{Governing Equations}

The equations solved are the conservative unsteady compressible two-dimensional axisymmetric Navier-Stokes equations

$$
\frac{\partial Q}{\partial t}+\frac{\partial F}{\partial x}+\frac{\partial G}{\partial y}=\frac{\partial F_{v}}{\partial x}+\frac{\partial G_{v}}{\partial y}+S
$$

where $Q$ is the solution flow field vector, $F$ and $G$ are the axial- and radial-direction inviscid flux vectors given by

$$
Q=\left[\begin{array}{c}
\rho \\
\rho u \\
\rho v \\
\rho E
\end{array}\right] \quad F=\left[\begin{array}{c}
\rho u \\
\rho u^{2}+p \\
\rho u v \\
(\rho E+p) u
\end{array}\right] \quad G=\left[\begin{array}{c}
\rho v \\
\rho v u \\
\rho v^{2}+p \\
(\rho E+p) v
\end{array}\right]
$$

and $F_{v}$ and $G_{v}$ are the axial- and radial-direction viscous and heat conduction flux vectors given by

$$
F_{v}=\left[\begin{array}{c}
0 \\
\tau_{x x} \\
\tau_{x y} \\
u \tau_{x x}+v \tau_{x y}-q_{x}
\end{array}\right] \quad G_{v}=\left[\begin{array}{c}
0 \\
\tau_{y x} \\
\tau_{y y} \\
u \tau_{y x}+v \tau_{y y}-q_{y}
\end{array}\right]
$$

The vector $S$ contains viscous flux and source terms associated with the axisymmetric geometry.

The source term, shear stresses and heat fluxes have the following form

$$
\begin{aligned}
& S=\frac{1}{y}\left\{\left[\begin{array}{c}
0 \\
\tau_{y x} \\
\tau_{y y}-\tau_{\theta \theta} \\
u \tau_{y x}+v \tau_{y y}-q_{y}
\end{array}\right]-\left[\begin{array}{c}
\rho v \\
\rho u v \\
\rho v^{2} \\
(\rho E+p) v
\end{array}\right]\right\} \\
& \tau_{x x}=\frac{2}{3} \frac{\mu}{\operatorname{Re}}\left(2 \frac{\partial u}{\partial x}-\frac{\partial v}{\partial y}-\frac{v}{y}\right) \quad \tau_{y y}=\frac{2}{3} \frac{\mu}{\operatorname{Re}}\left(2 \frac{\partial v}{\partial y}-\frac{\partial u}{\partial x}-\frac{v}{y}\right) \\
& \tau_{\theta \theta}=\frac{2}{3} \frac{\mu}{\operatorname{Re}}\left(2 \frac{v}{y}-\frac{\partial u}{\partial x}-\frac{\partial v}{\partial y}\right) \quad \tau_{x y}=\frac{\mu}{\operatorname{Re}}\left(\frac{\partial u}{\partial y}+\frac{\partial v}{\partial x}\right) \\
& q_{x}=-\frac{\gamma}{\gamma} \frac{q_{y}}{(\gamma-1) \operatorname{Pr} \operatorname{Re}} \frac{-\frac{\partial T}{\partial x}}{(\gamma-1) \operatorname{Pr} \operatorname{Re} \frac{\partial T}{\partial y}}
\end{aligned}
$$

A singularity exists along the axis of symmetry $y=0$. After applying L'Hopital rule and taking limit as $y$ goes to zero using the symmetry conditions $\frac{\partial u}{\partial y}=\frac{\partial \rho}{\partial y}=\frac{\partial T}{\partial y}=\frac{\partial p}{\partial y}=0, v=0$ at $\mathrm{y}=0$, source term along the axis $y=0$ becomes 


$$
S=\left[\begin{array}{c}
\left.\rho \frac{\partial v}{\partial y}\right|_{y=0} \\
\left.\rho u_{y=0} \frac{\partial v}{\partial y}\right|_{y=0}-\frac{\mu}{\operatorname{Re}}\left[\frac{\partial}{\partial y}\left(\left.\frac{\partial u}{\partial y}\right|_{y=0}+\left.\frac{\partial v}{\partial x}\right|_{y=0}\right)\right] \\
-\frac{\mu}{\operatorname{Re}} \frac{\partial}{\partial y}\left(\left.\frac{\partial v}{\partial y}\right|_{y=0}\right) \\
{\left[\left.(\rho e+p) \frac{\partial v}{\partial y}\right|_{y=0}-u_{y=0} \frac{\mu}{\operatorname{Re}}\left[\frac{\partial}{\partial y}\left(\left.\frac{\partial u}{\partial y}\right|_{y=0}+\left.\frac{\partial v}{\partial x}\right|_{y=0}\right)\right]-\left.\frac{\partial v}{\partial y}\right|_{y=0} \frac{2}{3} \frac{\mu}{\operatorname{Re}}\left(\left.\frac{\partial v}{\partial y}\right|_{y=0}-\left.\frac{\partial u}{\partial x}\right|_{y=0}\right)-\frac{\gamma}{(\gamma-1) \operatorname{Pr} \operatorname{Re} \frac{\partial}{\partial y}\left(\left.\frac{\partial T}{\partial y}\right|_{y=0}\right)}\right]}
\end{array}\right]
$$

Applying same procedure to shear stresses and heat fluxes we get a new term without a problem at $\mathrm{y}=0$.

$$
\begin{array}{ll}
\tau_{x x}=\frac{2}{3} \frac{\mu}{\operatorname{Re}}\left[\left.2\left(\frac{\partial u}{\partial x}-\frac{\partial v}{\partial y}\right)\right|_{y=0}\right] & \tau_{y y}=\left.\frac{2}{3} \frac{\mu}{\operatorname{Re}}\left(\frac{\partial v}{\partial y}-\frac{\partial u}{\partial x}\right)\right|_{y=0} \\
\tau_{\theta \theta}=\left.\frac{2}{3} \frac{\mu}{\operatorname{Re}}\left(\frac{\partial v}{\partial y}-\frac{\partial u}{\partial x}\right)\right|_{y=0}=0 \\
q_{x}=-\left.\frac{\gamma}{(\gamma-1) \operatorname{Pr} \operatorname{Re}} \frac{\partial T}{\partial x}\right|_{y=0} & q_{y}=0
\end{array}
$$

Here $(x, y)$ are the two-dimensional and $(x, y, \theta)$ are the axisymmetric coordinates and $(u, v)$ are the corresponding velocity components, $\rho$ is the density, $p$ is the pressure. $E$ is the total energy given by

$$
\begin{aligned}
& E=e+\frac{u^{2}+v^{2}}{2} \\
& e=c_{v} T, \quad p=\rho R T
\end{aligned}
$$

Here $e$ is the molecular internal energy and $T$ is the temperature.

The viscosity $(\mu)$ is computed using Sutherland's law and the coefficient of conductivity $(k)$ is given in terms of Prandtl number $(P r)$. The variables $\rho, p, T$ and velocity are non-dimensionalised by their corresponding reference variables $\rho_{\infty}, p_{\infty}, T_{\infty}$ and $\sqrt{R T}$ respectively. The reference value for length is computed by $\sqrt{v x_{0} / U_{\infty}}$, where $x_{0}$ is the location of the beginning of the computational domain in the stream wise direction. For the computation, the equations are transformed from physical coordinate system $(x, y)$ to the computational curvilinear coordinate system $(\xi, \eta)$ in a conservative manner and the governing equations become

$$
\frac{\partial \bar{Q}}{\partial t}+\frac{\partial \bar{F}}{\partial \xi}+\frac{\partial \bar{G}}{\partial \eta}=\frac{\partial \bar{F}_{v}}{\partial \xi}+\frac{\partial \bar{G}_{v}}{\partial \eta}+\bar{S}
$$

The components of the flux in the computational domain related to the flux in the physical domain by

$$
\bar{Q}=\frac{Q}{J}, \bar{F}=\frac{J}{|J|} F, \quad \bar{G}=\frac{J}{|J|} G, \quad \bar{F}_{v}=\frac{J}{|J|} F_{v}, \quad \bar{G}_{v}=\frac{J}{|J|} G_{v}, \bar{S}=\frac{J}{|J|} S \text { and } J=\left[\frac{\partial(\xi, \eta)}{\partial(x, y)}\right]
$$




\section{Solution Algorithm}

The governing equations are solved using 5th order accurate weighted essentially non-oscillatory (WENO) scheme for space discretization and using third order total-variation-diminishing (TVD) Runge-Kutta scheme for time integration. These methods are suitable in flows with discontinuities or high gradient regions. These schemes solve the governing equations discretely in a uniform structured computational domain in which flow properties are known at the grid nodes. WENO scheme approximate the spatial derivatives in a given direction to a higher order at the nodes, using the neighboring nodal values in that direction. TVD-RK scheme integrates the resulting equations in time to get the point values as a function of time. Since the spatial derivatives are independent of the coordinate directions, the method can easily add other dimensions. It is well known that approximating a discontinuous function by a higher order (two or more) polynomial generally introduces oscillatory behavior near the discontinuity, and this oscillation increases with the order of the approximation. The essentially non oscillatory (ENO) and the improvement of these WENO methods are developed to keep the higher order approximations in the smooth regions and to eliminate or suppress the oscillatory behavior near the discontinuities. They are achieved by systematically adopting or selecting the stencils based on the smoothness of the function, which is being approximated. Shu ${ }^{18}$ explains the WENO and the TVD methods and the formulas. Atkins ${ }^{19}$ gives the application of ENO method to the N-S equations. Balakumar ${ }^{20}$ describes in detail the solution method implemented in this computation.

At the outflow boundary, extrapolation boundary condition is used. At the wall, the simulation uses viscous conditions for the velocities and an adiabatic temperature condition, and it computes density from the continuity equation. In the mean flow computations, the simulation prescribes the free-stream values at the outer boundary, which lies outside the bow shock. The blunt cone is assumed to align with the free stream. In the unsteady computations, it superimposes the acoustic perturbations to the uniform mean flow at the upper boundary. The procedure is to first compute the steady mean flow by performing unsteady computations using a variable time step until the maximum residual reaches a small value $\sim 10^{-11}$. These computations use a CFL number of 0.2 . The next step is to introduce unsteady disturbances at the upper boundary of the computational domain and to perform time accurate computations to investigate the interaction and evolution of these disturbances to downstream. Since we use very fine spatial grid to resolve the leading edge region, these computations require very small time step which is taken as the minimum time step allowable for a CFL number of 0.2 .

The grid is generated using analytical formulae. The grid stretches in the $\eta$ direction close to the wall and is uniform outside of the boundary layer. In the $\xi$ direction, the grid is symmetric about the leading edge and very fine near the nose and is uniform in the flat region. The outer boundary that lies outside the shock follows a parabola so that the boundary layer growth could be captured accurately. The computational domain extends from $x=-0.015$ to 36.0 inches in the axial direction in this computation depending on the bluntness. Calculations were performed using a grid which has 32 blocks and each block has $127 \times 256$ grid points. Computational domain has approximately 1 million grid points. Due to the very fine grid requirement near the nose, the allowable time step is very small and the computations become very expensive to simulate the unsteady computations in the entire domain.

The acoustic field that impinges on the outer boundary is taken to be in the following form.

$$
p^{\prime}=\operatorname{Real}\left\{\tilde{p} e^{i \alpha_{a c} x \pm i \varepsilon_{a c} y-i \omega t}\right\}
$$

Here $\alpha_{a c}, \varepsilon_{a c}$ are the $x, y$ wave numbers, respectively, of the acoustic wave and $\omega$ is the corresponding frequency of the acoustic disturbance. The incident angle $\theta$ of the acoustic wave is defined as

$$
\theta=\tan ^{-1} \frac{\varepsilon_{a c}}{\alpha_{a c}}
$$

and in this paper computations are performed for zero incidence angles.

\section{Results}

Computations are performed for hypersonic flows at a free stream Mach number of 6.0 over a 5-degree halfangle cone with blunt leading edges. The flow parameters are given in Table 1 and the boundary layer edge conditions for a sharp cone are given in Table 2. Fig. 1 shows the schematic diagram of the computational set up. The nose region of the cone is modeled as a circle of the form

$$
\left(x-r_{0}\right)^{2}+y^{2}=r_{0}^{2} \text {. }
$$


Here $r_{0}$ is the nose radius. Simulations are performed for nose radii $r_{0}=0.001,0.05$ and 0.10 inches at a unit Reynolds numbers $7.8^{*} 10^{6} / \mathrm{ft}$. This provides nose Reynolds numbers of $650,32,500$ and 65,000 . To achieve higher nose Reynolds numbers, two computations are performed at a higher unit Reynolds number of $15.6 * 10^{6} / \mathrm{ft}$ for the nose radii of $r_{0}=0.05$ and 0.10 inches. Different cases are summarized in Table 3.

Table 1. Flow parameters for Horvath's ${ }^{13}$ wind tunnel model.

\begin{tabular}{|c|c|c|}
\hline Free stream & 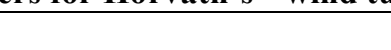 & \\
\hline Mach number & $\mathrm{M}_{\infty}=$ & 6.0 \\
\hline Unit Reynolds number & $\mathrm{Re}_{\infty}=$ & $7.8 \times 10^{6} / \mathrm{ft}$ \\
\hline Density & $\mathrm{P}_{\infty}=$ & $7.059 \times 10^{-3} \mathrm{lbm} / \mathrm{ft}^{3}$ \\
\hline Velocity & $\mathrm{U}_{\infty}=$ & $3140.21 \mathrm{ft} / \mathrm{s}$ \\
\hline Reservoir Pressure & $\mathrm{P}_{0}=$ & $475 \mathrm{psi}$ \\
\hline Reservoir Temperature & $\mathrm{T}_{0}=$ & $475^{\circ} \mathrm{F}$ \\
\hline Wall temperature & Adiabatic condition & \\
\hline Prandtl number & $\operatorname{Pr}=$ & 0.70 \\
\hline Ratio of specific heats & $\gamma=$ & 1.4 \\
\hline Length scale $\left(\mathrm{x}_{0}=0.5\right.$ inch $)$ & $\sqrt{v_{\infty} x_{0} / U_{\infty}}=$ & $7.30882 \times 10^{-5} \mathrm{ft}$ \\
\hline $\begin{array}{l}\text { The non-dimensional frequency } F \text { is } \\
\text { defined as }\end{array}$ & $F=\frac{2 \pi v_{\infty} f}{U_{\infty}^{2}}$ & $F=1.0 * 10^{-4} \cdot f=390 \mathrm{kHz}$ \\
\hline Local Reynolds number $\sqrt{\operatorname{Re}_{x}}$ & $\sqrt{\frac{U x}{v}}$ & \\
\hline
\end{tabular}

Table 2. Parameters along the cone surface.

\begin{tabular}{|l|c|}
\hline (Var.) edge $_{\text {( }}$ (Var.) $)_{\infty}$ & Sharp Cone \\
\hline Mach Number & 0.932 \\
\hline Pressure Ratio & 1.560 \\
\hline Density Ratio & 1.372 \\
\hline Temperature Ratio & 1.137 \\
\hline
\end{tabular}

Table 3. Parameters in the computations.

\begin{tabular}{|c|c|c|}
\hline $\begin{array}{c}\text { Nose radius } \\
\mathrm{r}_{0} \text { in. }\end{array}$ & $\begin{array}{c}\text { Unit } \\
\text { Reynolds } \\
\text { number } / \mathrm{ft}\end{array}$ & $\begin{array}{c}\text { Reynolds number } \\
\text { based on nose } \\
\text { radius }\end{array}$ \\
\hline 0.001 & $7.8^{*} 10^{6}$ & 650 \\
\hline 0.05 & $7.8^{*} 10^{6}$ & 32,500 \\
\hline 0.10 & $7.8^{*} 10^{6}$ & 65,000 \\
\hline 0.05 & $15.6^{*} 10^{6}$ & 65,000 \\
\hline 0.10 & $15.6^{*} 10^{6}$ & 130,000 \\
\hline
\end{tabular}




\section{Linear Stability}

Linear stability results for the similarity boundary layer over the cone using the inviscid conditions at the surface are presented in Fig. 2. Figure 2(a) shows the neutral stability diagram in $\left(\sqrt{ } R e_{x}, F\right)$ and $\left(\sqrt{ } e_{x}, \alpha\right)$ planes for twodimensional disturbances. The figure shows the first and second mode unstable regions and the variation of the wave number with the Reynolds number. Figure 2(b) shows the N-Factor curves for different frequencies. Here the variables are non-dimensionalized by the variables at the edge of boundary layer. To obtain the variables nondimensionalized by the free stream values as given in Table 1, the variables in this section should be multiplied by the appropriate factors from Table 2. The frequency variable $F$ has to be multiplied by 1.174 to obtain the values in terms of free stream values.

The neutral stability curve clearly shows the unstable first and the second mode regions for the boundary layers over the cone at a free stream Mach number 6.0. The first mode and the second mode neutral stability curves merge at a Reynolds number of $\sqrt{ } R_{x}=1600$ for the cone. The transition Reynolds numbers based on an N-Factor of 10 are about 3750 and the most amplified frequency is about $F=0.85 \times 10^{-4}$.

\section{Mean Flow}

Figure 3 shows the mean flow density contours computed using the WENO code. The figures $3(\mathrm{a}-\mathrm{e})$ show the results for the 5-degree half-angle cone at different nose radii $r_{0}=0.001,0.05,0.10$ and 0.10 (at two-times the unit Reynolds number) inches. Smaller nose radii cases $r_{0}=0.001,0.05$ and 0.10 are performed at a unit Reynolds number of $7.8 * 10^{6} / \mathrm{ft}$ which yield Reynolds numbers based on the nose radius of 650 to 65,000 . Figure 3(e) shows the results obtained at a higher unit Reynolds number of $15.6^{*} 10^{6} / \mathrm{ft}$ with $r_{0}=0.10$ which yields the Reynolds number based on the nose radius of 130,000. Figure 3(a) shows the density contours in larger domain and other figures show the flow field near the nose region. One interesting observation is that the inviscid density contours and the shock locations are same between Figs. 3(d) and (e) which are obtained with the same bluntness $r_{0}=0.10$ but at different unit Reynolds numbers 7.8 and $15.6 * 10^{6}$. The leading edge shocks are located approximately at 0.0002 , 0.008 and 0.016 in. upstream of the leading edge. The density profiles at different axial locations are plotted in Fig. 4(a)-(d) for the different bluntness cases $r=0.001,0.05,0.05\left(\operatorname{Re}_{\alpha} / \mathrm{ft}=15.6 * 10^{6}\right)$ and 0.10 inches in the similarity coordinates. The compressible Blasius similarity profile is also included for comparison and Fig. 4(d) and (e) show the profiles for $r_{0}=0.05$ and 0.10 inches in the physical coordinate. With increasing bluntness, the profiles did not approach the similarity profiles within the computational domain, which is closer to the transition onset point. The difference between the profiles with the bluntness and the similarity profiles increases with the bluntness.

Figures 5(a)-(d) depict the entropy contours for different bluntness cases $r_{0}=0.001,0.05,0.10$ and 0.10 $\left(\operatorname{Re}_{\alpha} / \mathrm{ft}=15.6^{*} 10^{6}\right)$ inches. For the small bluntness case $r_{0}=0.001$, only the boundary layer appears near the nose region and the entropy layer is not discernable in the outer part of the boundary layer. For the higher bluntness cases the entropy layer that is visible near the nose region persists downstream and eventually merges with the boundary layer. Figures 6(a)-(d) show the entropy profiles at different axial locations for different bluntness cases. As was observed in the contours any discernable entropy layer appears for the small bluntness case. For the bluntness case $r_{0}=0.05$, two layers are clearly seen in the profiles at $x=0.50$ and 1.0 inches. One layer is very close to the wall with large gradient and the other is away from the wall with a small gradient. The outside entropy layer merges with the boundary layer near the wall close to $x=2.0$ inches. At higher bluntness cases, the two layers are more evident and the merging occurs at larger distances from the nose. For the bluntness case $r_{0}=0.10$, the two layers merge close to $x=6.0$ inches and for the case $r_{0}=0.10$ with larger unit Reynolds number the merging occurs close to $x=6.0$ inches which is equivalent to 12.0 inches when it is converted to the same unit Reynolds number.

Figures 7(a) and (b) show the N-Factors and the growth rates for the most amplified disturbances computed using the mean profiles obtained from the numerical simulation for different bluntness $r_{0}=0.001,0.05,0.10$ with the unit Reynolds number of $7.8^{*} 10^{6} / \mathrm{ft}$ and for $r_{0}=0.05,0.10$ with the higher unit Reynolds number of $15.6^{*} 10^{6} / \mathrm{ft}$. For comparison, the results for the Blasius similarity profiles, which model a sharp leading edge, are also shown in the figure. The frequency for the most amplified wave is about $F=0.90^{*} 10^{-4}$ for the similarity profiles. There is significant difference both for the mean flow and the stability results with increasing bluntness. The growth rates become smaller and the N-Factor curves move downstream. For the smaller bluntness $r_{0}=0.001$, the N-Factor curve remains closer to the similarity curve. For the larger bluntness cases the growth rates become smaller and the NFactor curves move further to the right. The most amplified frequencies are $\left(0.85^{*} 10^{-4}, 0.75^{*} 10^{-4}, 0.60^{*} 10^{-4}\right.$, $0.425^{*} 10^{-4}$ ) for $r_{0}=0.001,0.05,0.10$ and 0.10 (with higher unit Reynolds number) inches respectively. This shows that the frequencies of the most amplified disturbances become smaller with increasing bluntness. The growth rate 
curves are similar to the Blasius profile for all the cases. The transition Reynolds numbers obtained using the Nfactor of 10.0 for different bluntness cases are summarized in Table 5 and plotted in Fig. 8. The ratio between the transition Reynolds number with bluntness and the transition Reynolds number for the similarity profile,

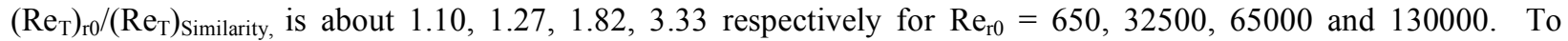
differentiate between the effects of free stream unit Reynolds number and the nose Reynolds number one simulation is performed for the bluntness case $r_{0}=0.05$ at twice the unit Reynolds number of $2 * 7.8^{*} 10^{6} / \mathrm{ft}$. The expectation is that the results from this simulation should be close to the results obtained for the case with $r_{0}=0.10$ at a unit Reynolds number of $7.8 * 10^{6} / \mathrm{ft}$. It is interesting to see in Fig. 7 that the growth rates and the N-Factor curves are very close to each other and the most amplified non-dimensional frequencies are also the same $F=0.60^{*} 10^{-4}$. The transition Reynolds numbers are $21.90 * 10^{6}$ and $23.70 * 10^{6}$ for the cases $\mathrm{r}_{0}=0.10$ and 0.05 (higher unit Reynolds number) respectively. The transition Reynolds number for this case is also included in Fig. (8). This implies that the stability and the transition over blunt bodies are determined by the nose Reynolds number. Previous experiments ${ }^{1}$ and the stability calculations ${ }^{3}$ showed that the transition Reynolds number for a blunt cone at a Mach number of 8 with nose Reynolds numbers of 30,000 increased by a factor of 1.7 2.0 compared to a sharp cone. Potter ${ }^{5}$ found from a series of aeroballistic range experiments on nominally sharp cones that the transition Reynolds number increases with the free stream unit Reynolds number as a power of 0.63 . A line with the slope of 0.60 is included in Fig. (8) for comparison. The prediction from the present calculations follows this slope closely.

Table 5. Transition Reynolds number for the blunt cones.

\begin{tabular}{|c|c|c|c|c|}
\hline $\begin{array}{c}\text { Nose radius } \\
r_{0} \text { in. }\end{array}$ & $\begin{array}{c}\text { Reynolds number } \\
\text { based on nose } \\
\text { radius }\end{array}$ & $\begin{array}{c}\text { Transition } \\
\text { location } X(\text { in. })\end{array}$ & $\begin{array}{c}\text { Transition } \\
\text { Reynolds number } \\
* 10^{6}\end{array}$ & $\begin{array}{c}\text { Ratio } \\
\left(\operatorname{Re}_{T}\right)_{\text {ro }} /\left(\operatorname{Re}_{T}\right)_{\text {Similarity }}\end{array}$ \\
\hline 0.0 & 0 & 18.55 & 12.06 & 1.00 \\
\hline 0.001 & 650 & 20.50 & 13.32 & 1.10 \\
\hline 0.05 & 32,500 & 23.50 & 15.30 & 1.27 \\
\hline 0.10 & 65,000 & 33.70 & 21.90 & 3.33 \\
\hline $0.10(2 * 7.8)$ & 130,000 & 61.88 & 40.22 & 1.96 \\
\hline $0.05(2 * 7.8)$ & 65,000 & 36.44 & 23.70 & \\
\hline
\end{tabular}

\section{Interaction of Slow Acoustic Waves with the Boundary Layer}

After the mean flow is computed two dimensional slow acoustic disturbances are introduced at the outer computational boundary and the time accurate simulations are performed. Unsteady simulation results are presented for the cases $r_{0}=0.001,0.05,0.05\left(2 * \operatorname{Re}_{\alpha}\right), 0.10$ inches at the most amplified frequencies $F=0.80 \times 10^{-4}, 0.75 \times 10^{-4}$, $0.60 \times 10^{-4}$ and $0.60 * 10^{-4}$. These frequencies correspond to $304,285,228$ and $556 \mathrm{kHz}$ respectively for these unit Reynolds numbers. To remain in the linear regime, the amplitude of the forcing freestream acoustic waves is given a small value of $p_{a c} / p_{\infty}=2 * 10^{-6}$. Even with this small amplitude, nonlinearity starts to develop near the end of computational domain for the small bluntness case $r_{0}=0.001$ case with the frequency $F=0.80 \times 10^{-4}$.

Figures 9 (a) and (b) show the results for the evolution of unsteady fluctuations obtained from the simulations for the slow wave at a fixed time for the case $r_{0}=0.05$ and $F=0.75 \times 10^{-4}$. Figure 9 (a) shows the contours of the density fluctuations near the nose region up to $x \sim 6.0$ inches and Fig. 9(b) depicts the results near the end of the computational domain $x=15 \sim 25$ inches. Figures 10(a)-(d) display the expanded view of the density contours near the wall along the axial direction. The perturbation field can be divided into three regions. One region is the area outside the shock where the acoustic waves propagate uniformly. The second region is the shock layer across which the acoustic waves are transmitted. The third region is the area between the shock and the boundary layer. This region consists of transmitted external acoustic field and the disturbances that are radiated from the boundary layer. The figures also show that the flow field between the shock and the wall exhibit four different regions of excitations. 
One is the region directly below the shock where small wave diffraction occurs, second is the region below this diffraction zone and above the entropy layer where the disturbances are quieter, third is the entropy layer and the boundary layer edge region where large perturbations exist and fourth is the region near the wall. The first important observation is that near the nose region (Figs.9 (a), 10(a)) the acoustic disturbances propagate across the leading edge bow shock and perturb the entropy layer. These disturbances as they evolve downstream remain inside the entropy layer and get into the boundary layer further downstream. Figures 10(b)-(d) clearly show that these disturbances remain near the edge of the boundary layer for a long distance before they become the highly unstable second mode. Another interesting observation is that the region between the boundary layer and the shock layer is quieter compared to the acoustic waves outside the shock layer. This quiet region originates from the leading edge region (Figs 9(a), 10(a)) where the bow shock and the oblique shock meet. This implies that the acoustic waves are weakly transmitted through the shock and do not directly interact with the boundary layer further downstream. This was also observed in the flat plate simulation ${ }^{21}$ where as the acoustic wave incidence angle is increased disturbances become quiets in the windward side.

Figures 11(a)-(h) show the evolution of the wall pressure fluctuations for different cases. Figures 11(a), (c), (e), and (g) show the amplitude of the pressure fluctuations along the wall in a linear scale, while Figs. 11(b), (d), (f) and (h) depict the results in a log scale. This figure also includes the results from the parabolized stability equations (PSE) computations obtained for the same mean boundary layer profiles. The growth of the disturbances agrees very well with the PSE results. The figures clearly show the generation and the eventual exponential growth of the instability waves inside the boundary layer. First observation is that there are large differences in the amplitude levels of the disturbances attained between the small bluntness case and the large bluntness cases. In all the cases the amplitude of the free stream acoustic pressure is the same $p_{a c} / p_{\infty}=2 * 10^{-6}$. For the small bluntness case $r_{0}=0.001$, Figs. 11(a) and (b), the disturbances grow from the leading edge and reach large amplitude levels of 0.50 near the predicted transition onset point. The slow wave whose wavelength is closer to the wavelength of the instability wave transform into instability wave smoothly near the nose region. The parallel linear computations show that the first mode amplifies weakly up to $x \sim 10$ inch for this frequency and yields an N-Factor of 1.0 near $x \sim 10$. However, the simulation shows that the first mode disturbances are growing much stronger near the leading edge and yields an NFactor of 3.0 near $x \sim 10$. Hence, the non-parallel effects are stronger in the small bluntness case near the nose region. The maximum amplitudes obtained for the large bluntness cases $r_{0}=0.05,0.05\left(2 * \mathrm{Re}_{\alpha}\right), 0.10$ ( Figs. 11(c-d), (e-f), (gh)) are very small in the range of $\sim 10^{-4}$. The reason for this is the disappearance of the amplification of the first modes in the early part of the evolution. The disturbances not only grow but decay by two orders in magnitude before they start to grow due to the instability of second modes. This may be due to the thickening of the boundary layer due to the entropy layer.

Following the PSE results up to the neutral point, the initial amplitude of the instability waves at the neutral point can be estimated. From these values the receptivity coefficients defined by the amplitude of the pressure fluctuations at the wall at the neutral point non-dimensionalized by the free-stream acoustic pressure can be evaluated.

$$
C_{\text {recpt }, p_{\text {wall }}}=\frac{\left(p_{\text {wall }}\right)_{n}}{p_{a c}}
$$

Table 6 shows the amplitude of the pressure fluctuations $\left(p_{\text {wall }}\right)_{n}$ at the neutral point and the receptivity coefficients for different nose radii. The amplitudes are $8.5^{*} 10^{-6}$ for the small bluntness case and they are on the order of $10^{-9}$ for the large bluntness cases. This reflects in the magnitude of the receptivity coefficients. The receptivity coefficients are $4.23,1.85^{*} 10^{-3}$ and $4.75^{*} 10^{-3}$ for the cases the $=0.001,0.05$ and 0.10 inches. Another interesting observation is comparison of the amplitudes and the receptivity coefficients for the two cases $r_{0}=0.10$ and $r_{0}=0.05\left(2 * \operatorname{Re}_{\alpha}\right)$. The amplitudes and the receptivity coefficients are almost the same for these two cases. Hence it is not only the instability properties but the receptivity coefficients also depend only on the nose Reynolds number. This implies that unit Reynolds number effect is directly the consequence of the variation in the nose Reynolds numbers.

Figure 12 shows the density fluctuations inside the boundary layer near the nose region for a small $r_{0}=0.001$ and a large $r_{0}=0.10$ cases. This clearly shows the effect of bluntness in the generation of disturbances near the nose region. As was discussed previously, at small bluntness case there is no entropy layer and the disturbances excite the boundary layer up to the wall. In the large bluntness case, the disturbances are seen only away from the wall inside the entropy region. 
Table 6. Receptivity coefficients for different nose radii.

\begin{tabular}{|c|c|c|c|c|}
\hline $\begin{array}{c}\text { Nose radii } \\
r_{0} \text { (in.) }\end{array}$ & $\begin{array}{c}\text { Nose } \\
\text { Reynolds } \\
\text { number }\end{array}$ & $F\left(\mathrm{x} 10^{-4}\right)$ & $\left(p_{\text {wall }}\right)_{n}$ & $C_{\text {recpt }, p_{\text {wall }}, \text { Slow }}$ \\
\hline 0.001 & 650 & 0.80 & $8.5 * 10^{-6}$ & 4.23 \\
\hline 0.05 & 32,500 & 0.75 & $3.7 * 10^{-9}$ & $1.85 * 10^{-3}$ \\
\hline 0.10 & 65,000 & 0.60 & $9.5 * 10^{-9}$ & $4.75 * 10^{-3}$ \\
\hline $0.05(2 * \mathrm{RE})$ & 65,000 & 0.60 & $10.0 * 10^{-9}$ & $5.00 * 10^{-3}$ \\
\hline
\end{tabular}

\section{Discussion and Conclusion}

The receptivity and the stability of hypersonic boundary layers over a blunt cone with 5 degrees half-angle are numerically investigated at a free stream Mach number of 6.0 and at a Reynolds number of $7.8 \times 10^{6} / \mathrm{ft}$. Both steady and unsteady solutions are obtained by solving compressible Navier-Stokes equations using the $5^{\text {th }}$ order accurate weighted essentially non-oscillatory (WENO) scheme for space discretization and using a third-order total-variationdiminishing (TVD) Runge-Kutta scheme for time integration. Computations are performed for different nose radii $r_{0}=0.001,0.05,0.05\left(2 * \operatorname{Re}_{\alpha}\right), 0.10,0.10\left(2 * \operatorname{Re}_{\alpha}\right)$ inches which yields nose Reynolds numbers of $650,32500,65000$, $65000,130000$.

The results show that the bluntness has a strong stabilizing effect on the stability of the boundary layers. The transition Reynolds number increases slowly up to a nose Reynolds number of 30,000 and then increases sharply at higher nose Reynolds numbers. The transition Reynolds number for a cone at a nose Reynolds number of 65,000 is about 1.8 times larger than that for the Blasius boundary layer. This is due to the entropy layers that are generated near the leading edges. These layers persist for longer distances with increasing bluntness. There may be other unstable modes in the entropy layer as were observed in the experiments ${ }^{1}$ other than the first-mode type instabilities that were considered in this paper. Whether they exist and what role these waves play in the transition process still has to be investigated.

At small bluntness case, the disturbances grow starting from the nose region and reach very large values $\sim 0.50$ near the transition point. The growth of the first mode is much stronger for this case due to the non-parallel effects. The amplitude levels of the disturbances are much smaller in the order of $10^{-4}$ in the larger bluntness cases. There is no unstable first modes in the large bluntness cases and the disturbances decay by two orders before they start to grow due to the second mode instability. The receptivity coefficients at small bluntness $r_{0}=0.001$ inches is about 4.23 and they are much smaller in the order of $\sim 10^{-3}$ in the larger bluntness cases. This raises some questions about the transition process over blunt bodies. If the receptivity coefficients are very small for the second modes as was found in this paper, how can the amplitude of the disturbances attain high values. One possibility is that the NFactors are larger, about 15 16, in these cases. This will increase the transition Reynolds number further than that is computed in this paper. Another possibility is that the receptivity coefficients at non-zero acoustic incident angles may be larger than is obtained at zero incident angles. These computations have to be carried out to find the largest receptivity coefficients.

\section{Acknowledgement}

The first and the third authors are supported by Flow Physics and Control Branch of NASA Langley Research Center for this work. 


\section{References}

Periodicals

${ }^{1}$ Stetson, K. F., Thompson, E. R., Donaldson, J. C., and Siler, L. G., 1984, Laminar Boundary Layer Stability Experiments on a Cone at Mach 8, Part 2: Blunt Cone, AIAA Paper, 0006.

${ }^{2}$ Reshotko, E. and Khan, M. M. S., September 1979, Stability of the Laminar Boundary Layer on a Blunted Plate in Supersonic Flow, IUTAM Laminar-Turbulent Transition Symposium, Stuttgart, F.R.G.

${ }^{3}$ Malik, M. R., Spall, R. E., and Chang, C. L., 1990, Effect of Nose Bluntness on Boundary Layer Stability and Transition, AIAA Paper, 0112.

${ }^{4} \mathrm{Hu}, \mathrm{S}$. E., and Zhong, X., 1998, Hypersonic Boundary-Layer Stability over Blunt Leading Edges with Bow-Shock Effects, AIAA Paper, 0433.

${ }^{5}$ Zhnog, X., and Ma, Y., 2002, Receptivity and Linear Stability of Stetson's Mach 8 Blunt Cone Stability Experiments, AIAA Paper, 2849.

${ }^{6}$ John, C. Evvard, Sept 5-13, 1956, Transition studies on blunted bodies at supersonic speeds, Presented at 9th International Congress of Applied Mechanics, Brussels.

${ }^{7}$ Stetson, K. F., 1983, Nosetip Bluntness Effects on Cone Frustum Boundary Layer Transition in Hypersonic Flow, AIAA Paper, 1763.

${ }^{8}$ Maslov, A. A., Shiplyuk, A. N., Sidorenko, A., and Arnal, D., 2001, Leading Edge Receptivity of a Hypersonic Boundary Layer on a Flat Plate, Journal of Fluid Mechanics, 426,73-94.

${ }^{9}$ Maslov, A. A., Mirinov, S. G., Shiplyuk, A. N., 2002, Hypersonic Flow Stability Experiments, AIAA Paper, 0153.

${ }^{10}$ Rufer, S. J., and Schneider, S. P., 2006, Hot-Wire Measurements of Instability Waves on Cones at Mach 6, AIAA Paper, 3054.

${ }^{11}$ Schneider, S. P., 2001, Hypersonic Laminar Instability on Round Cones Near Zero Angle of Attack, AIAA Paper, 0206.

${ }^{12}$ Schneider, S. P., 2004, Hypersonic Laminar-Turbulent Transition on Circular Cones and Scramjet Forebodies, Progress in Aerospace Sciences, Vol. 40, 1-50.

${ }^{13}$ Horvath, T. J., Berry, S. C., Hollis, B. R., Chang, C. C., Singer, B. A., 2002, Boundary Layer Transition On Slender Cones In Conventional And Low Disturbances Mach 6 Wind Tunnels, AIAA Paper, 2743

${ }^{14}$ Potter, J. L., 1974, Boundary Layer Transition on Supersonic Cones in an Aerobalistic Range, AIAA Journal, Vol. 13, No. 3,270-277.

${ }^{15}$ Rosenboom, I., Hein, S., and Dallmann, U., 1999, Influence of Nose Bluntness on Boundary Layer Instabilities in Hypersonic Cone Flows, AIAA Paper, 3591.

${ }^{16}$ Zhong, X., 2005, Effect of Nose Bluntness on Hypersonic Boundary Layer Receptivity over a Blunt Cone, AIAA Paper, 5022.

${ }^{17}$ Balakumar, P., 2006, Stability of Supersonic Boundary Layers over Blunt Wedges, AIAA Paper, 3053.

${ }^{18}$ Shu, C. W., 1997, Essentially Non-Oscillatory And Weighted Essentially Non-Oscillatory Schemes For Hyperbolic Conservation Laws, NASA/CR-97-206253 and ICASE Report No. 97-6.

${ }^{19}$ Atkins, H. L., 1991, High-Order ENO Methods for the Unsteady Compressible Navier-Stokes Equations, AIAA Paper, 1557.

${ }^{20}$ Balakumar, P., 2002, Stability of Hypersonic Boundary Layers Over a Compression Corner, AIAA Paper, 2848.

${ }^{21}$ Malik, M. R., and Balakumar, P., 2005, Receptivity of Supersonic Boundary Layers to Acoustic Disturbances, AIAA Paper, 5027. 


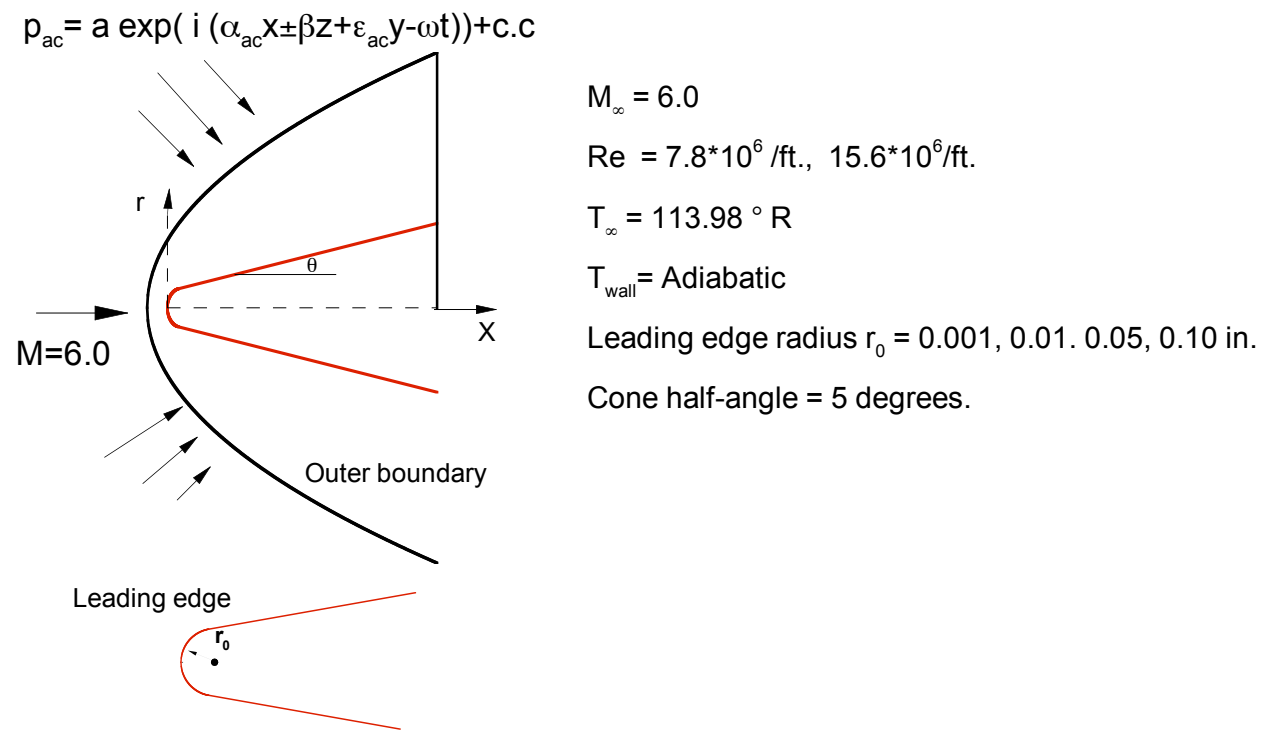

Figure 1. Schematic diagram of the computational model

(a)

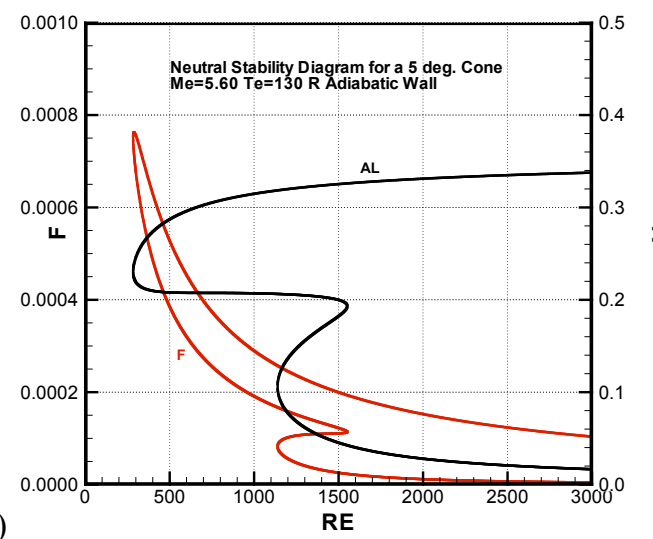

(b)

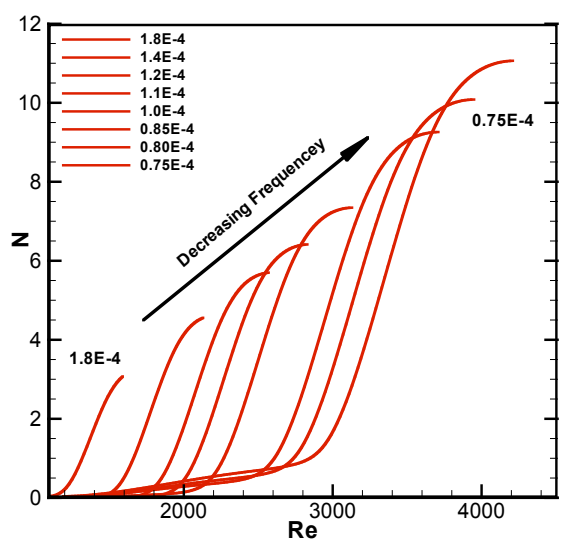

Figure 2. (a) Neutral stability diagram and variation of wave number with Reynolds number. (b) N-Factor curves for decreasing frequency for blunt cone. $r_{0}=0.001$ ". 

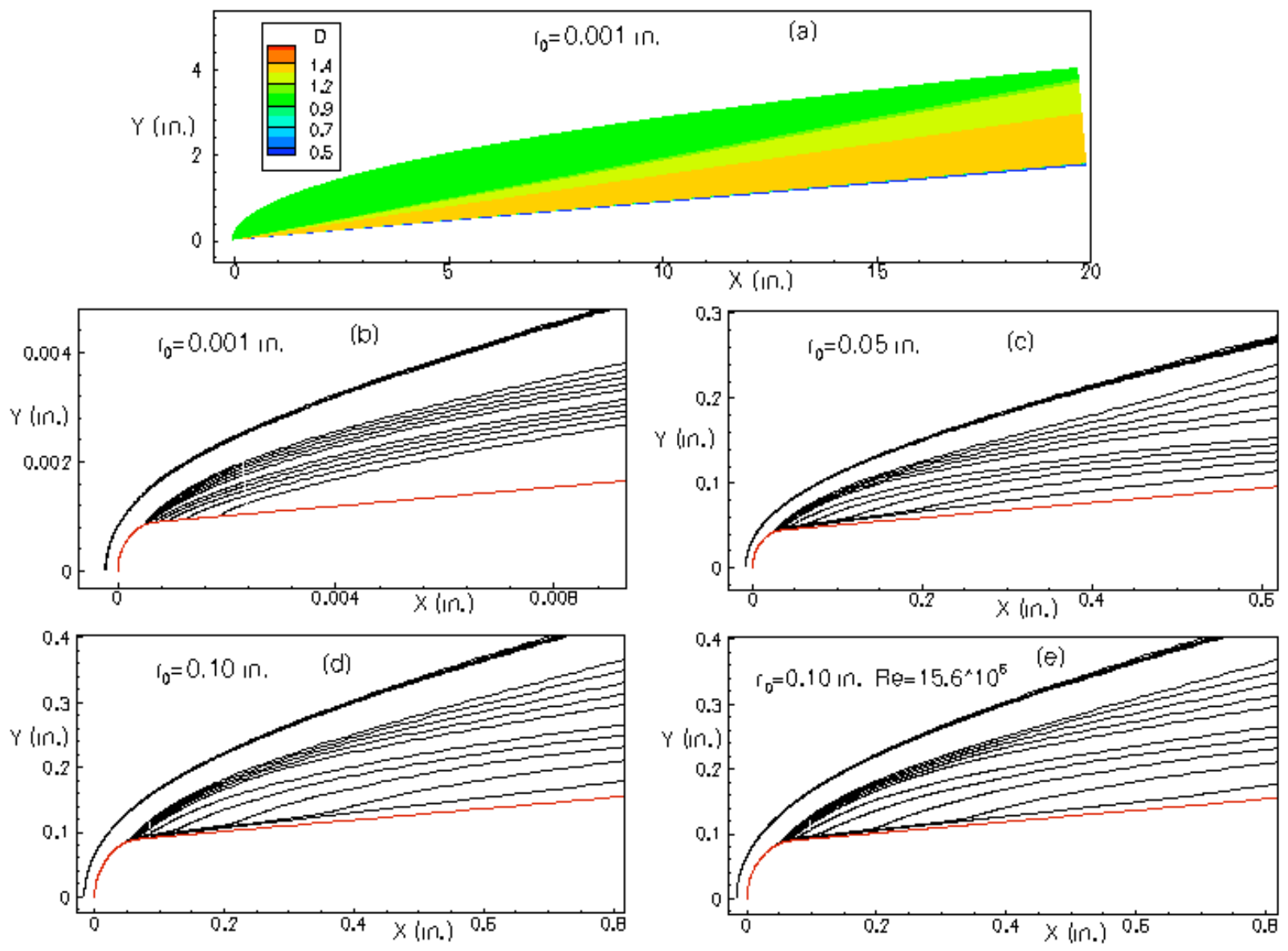

Figure 3. Contours of the density for flow over a cone with different bluntness at $M=6.0$. (a), (b) $r_{0}=0.001$ in. $R e=7.8 \times 10^{6}$, (c) $r_{0}=0.05$ in. $R e=7.8 \times 10^{6}$, (d) $r_{0}=0.10$ in. $R e=7.8 \times 10^{6}$, (e) $r_{0}=0.10$ in. $R e=15.6 \times 10^{6}$. 
(a) $r_{0}=0.001$ in.

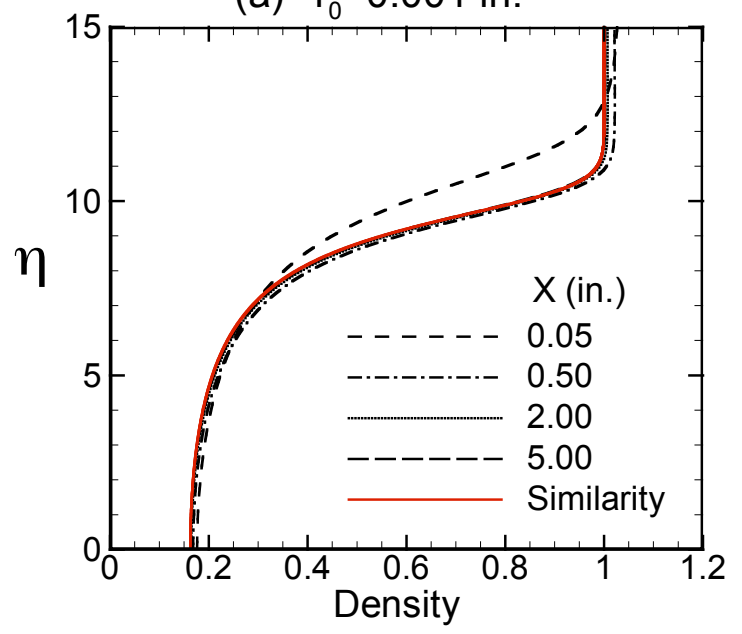

(c) $r_{0}=0.05$ in. $2 \times R E$

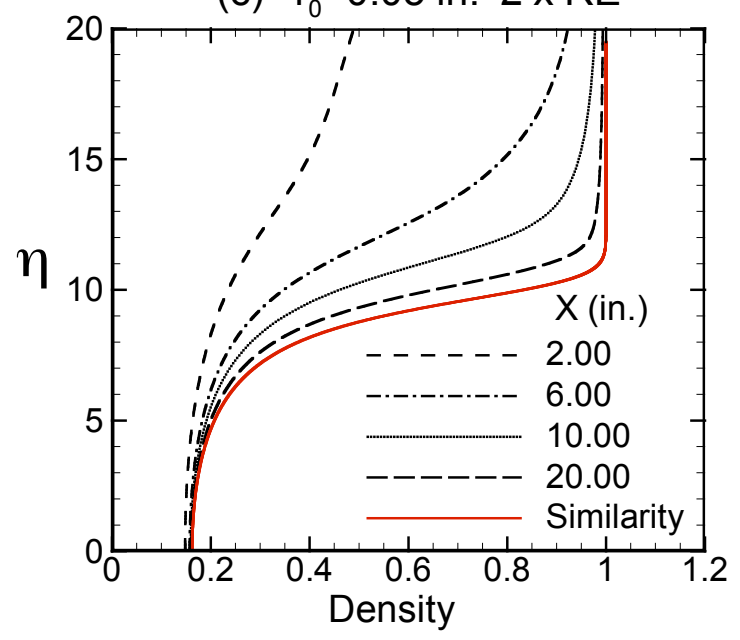

(e) $r_{0}=0.05$ in.

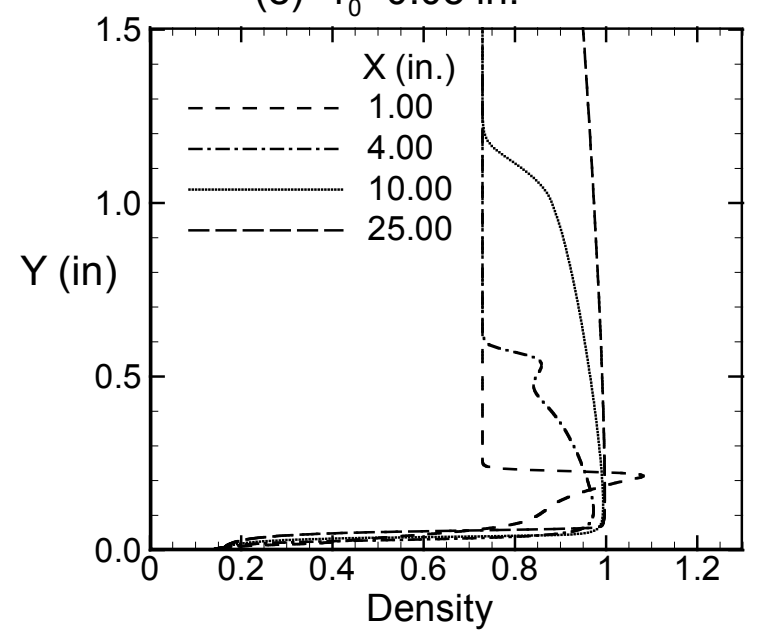

(b) $r_{0}=0.05$ in.

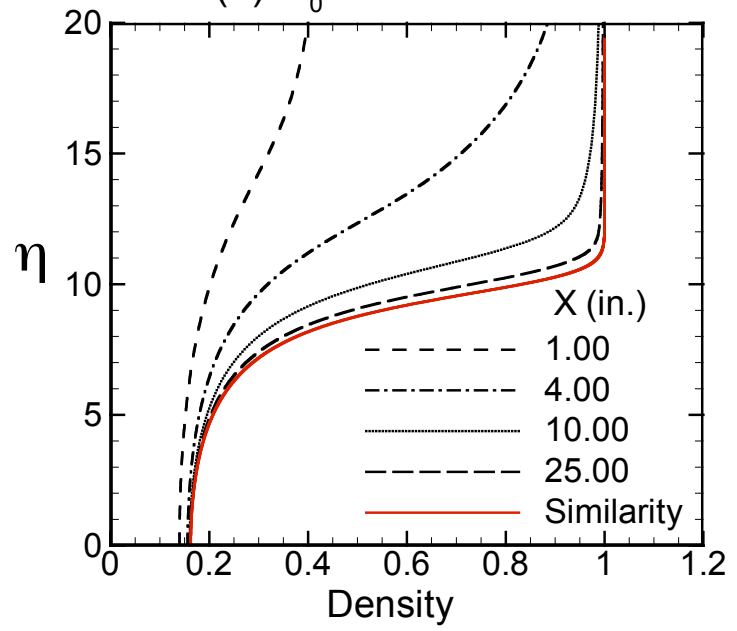

(d) $r_{0}=0.10$ in.

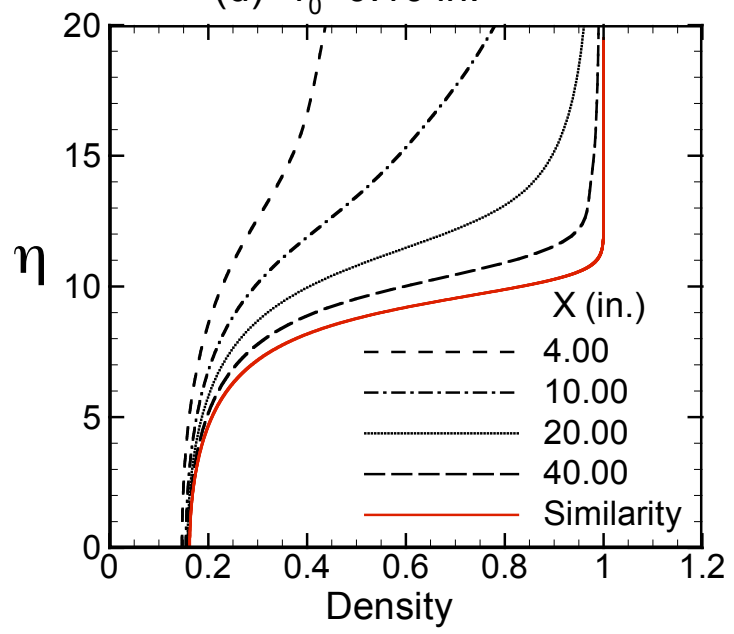

(f) $r_{0}=0.10 \mathrm{in}$.

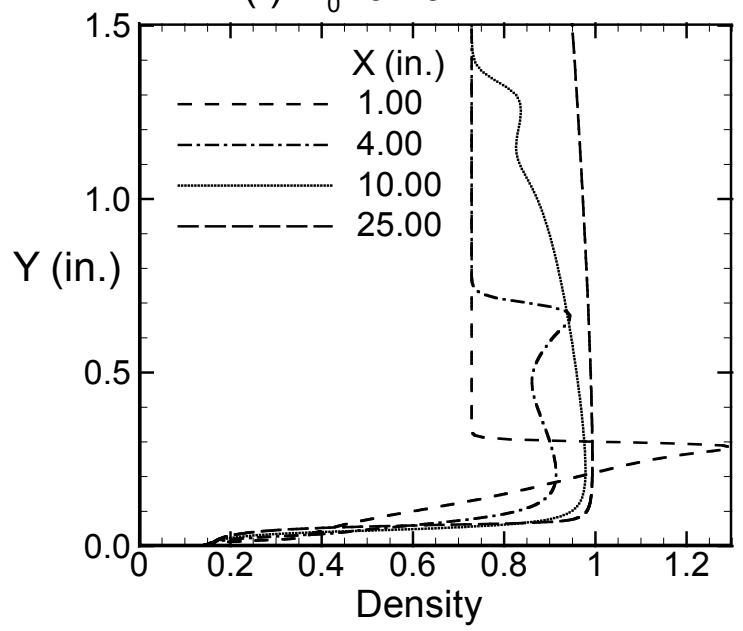

Figure 4. Mean density profiles at different $X$ locations using similarity coordinates for (a) $r_{0}=0.001 \mathrm{in}$. $R e=7.8 \times 10^{6}$, (b) $r_{0}=0.05$ in. $R e=7.8 \times 10^{6}$, (c) $r_{0}=0.05$ in. $R e=15.6 \times 10^{6}$, (d) $r_{0}=0.10$ in. $R e=7.8 \times 10^{6}$. Density profiles versus $Y$ coordinate for $(e) r_{0}=0.05$ in. $R e=7.8 \times 10^{6}$, (f) $r_{0}=0.10$ in. $R e=7.8 \times 10^{6}$. 

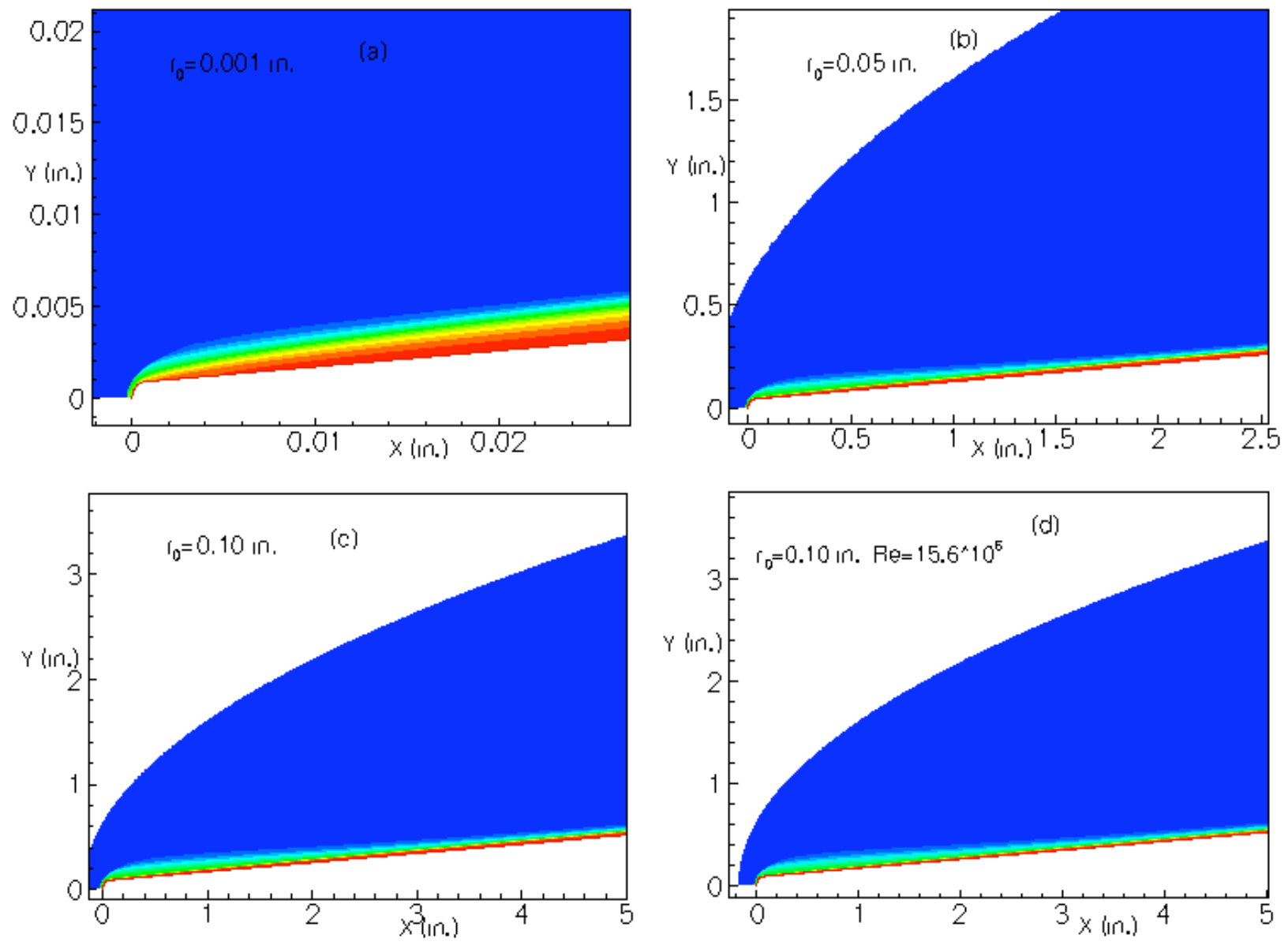

Figure 5. Contours of the entropy for flow over a cone with different bluntness at $M=6.0$. (a) $r_{0}=0.001$ in. $R e=7.8 \times 10^{6}$, (b) $r_{0}=0.05$ in. $R e=7.8 \times 10^{6}$, (c) $r_{0}=0.10$ in. $R e=7.8 \times 10^{6}$, (d) $r_{0}=0.10$ in. $R e=15.6 \times 10^{6}$. 
(a) $r_{0}=0.001 \mathrm{in}$.

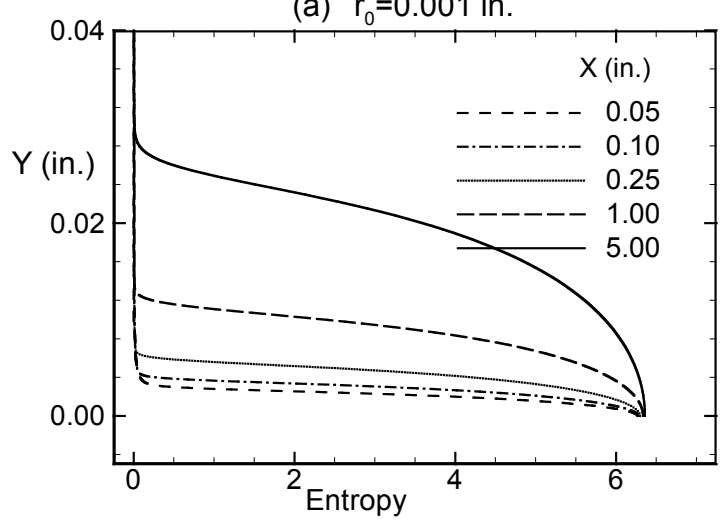

(c) $r_{0}=0.10 \mathrm{in}$.

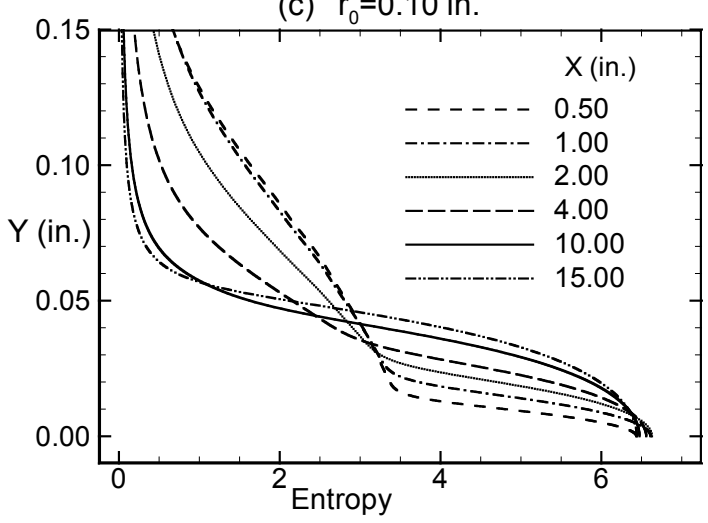

(b) $r_{0}=0.05$ in.

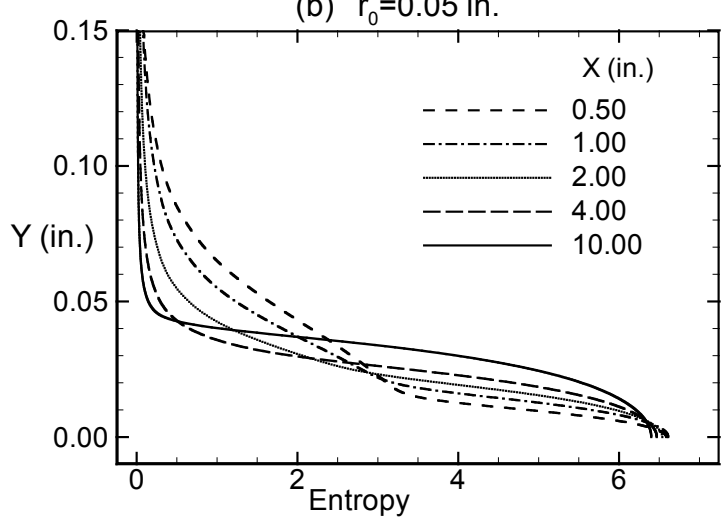

(d) $r_{0}=0.10$ in. $2 \times R E$

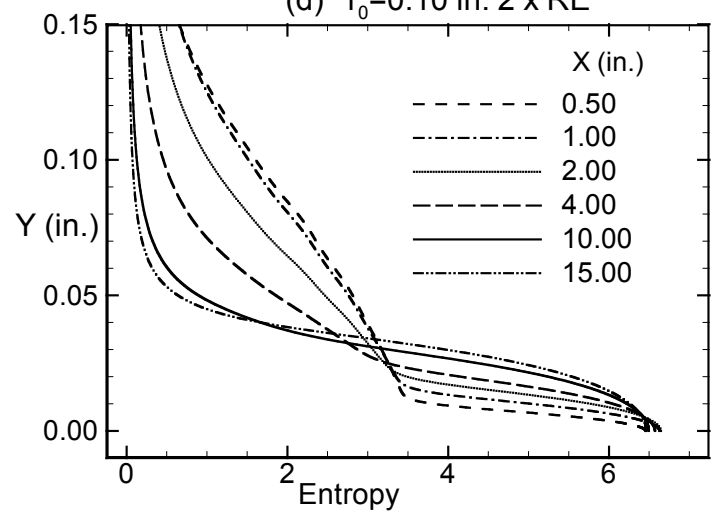

Figure 6. Entropy profiles at different $X$ stations for (a) $r_{0}=0.001 \mathrm{in}$. $R e=7.8 \times 10^{6}$, (b) $r_{0}=0.05 \mathrm{in}$. $R e=7.8 \times 10^{6}$, (c) $r_{0}=0.10$ in. $R e=7.8 \times 10^{6}$, (d) $r_{0}=0.10$ in. $R e=15.6 \times 10^{6}$.

(a)

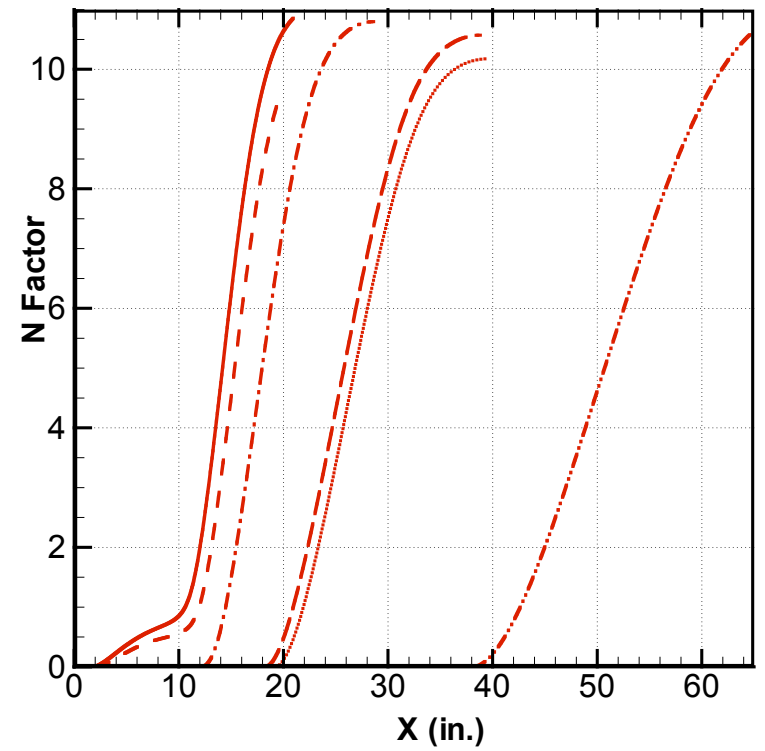

(b)

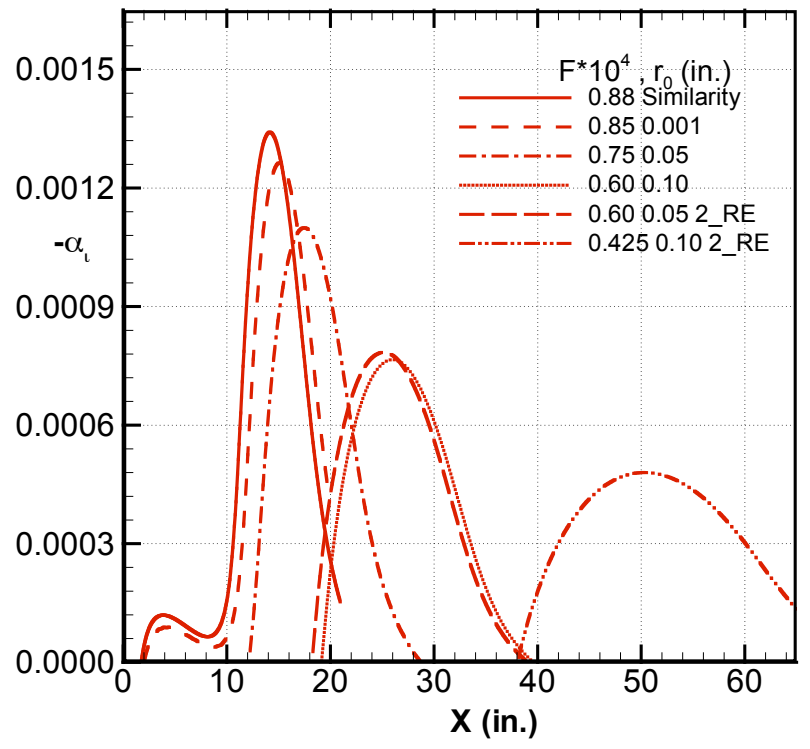

Figure 7. N-Factors (a) and growth rates (b) for the most amplified disturbances for $r_{0}=0.001,0.05,0.10$ in. 


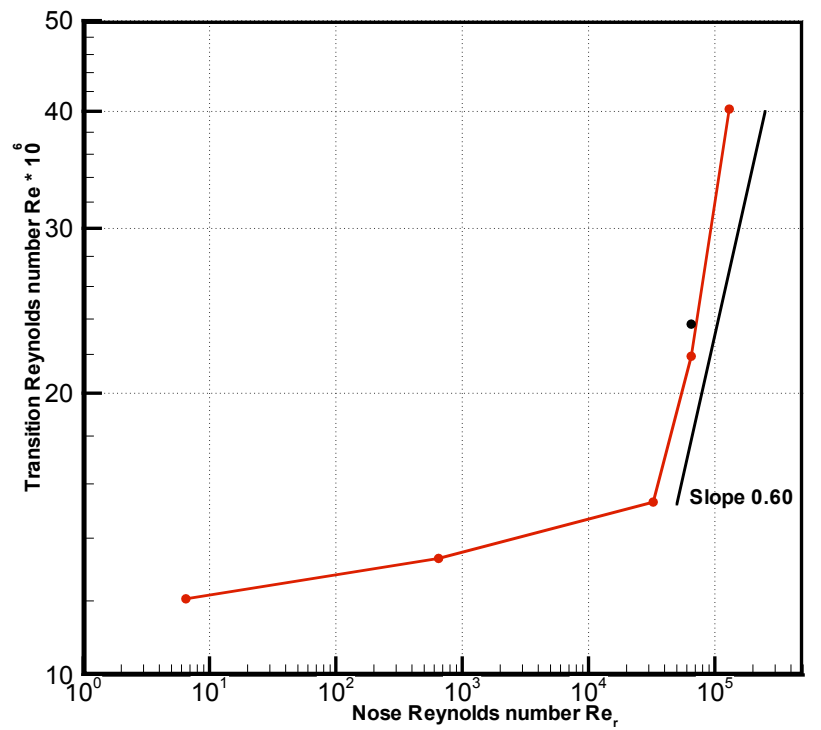

Figure 8. The transition Reynolds numbers for different bluntness.
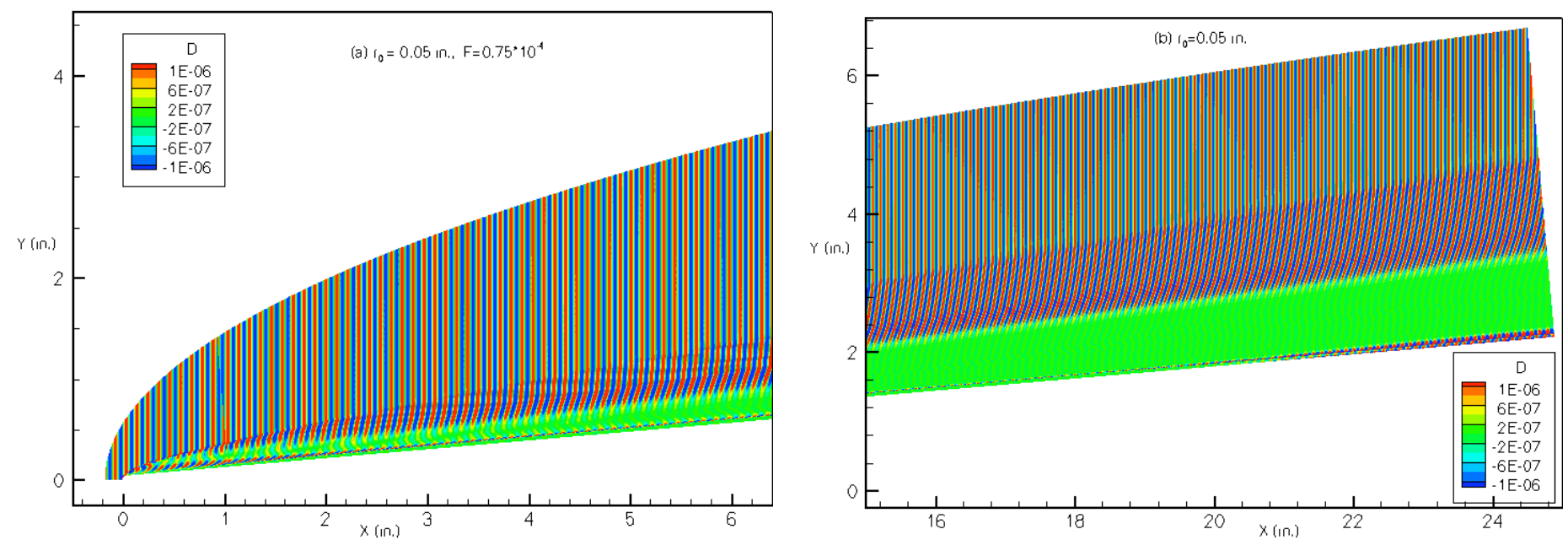

Figure 9. Contours of the unsteady density fluctuations due to the interaction of slow acoustic wave with a blunt cone: $F=0.75 \times 10^{-4}$. (a) Nose part. (b) Flat end. 


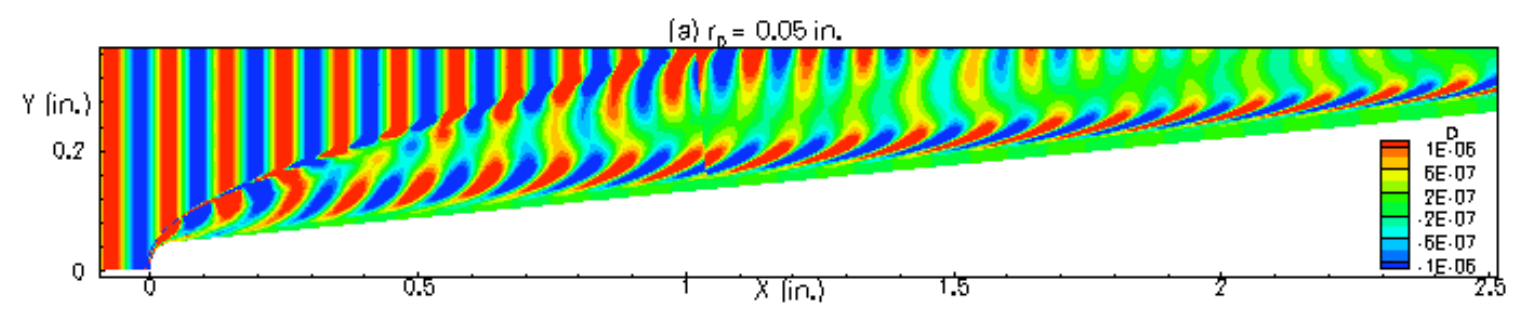

(b)

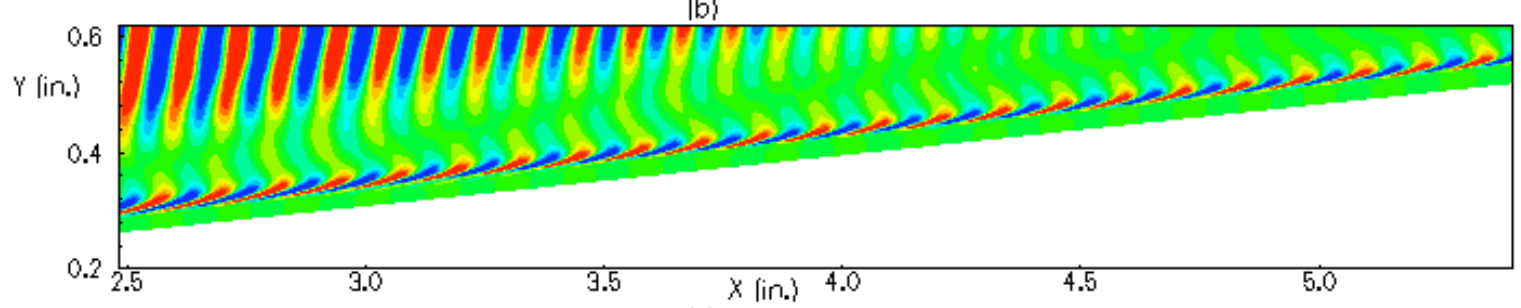

(c)

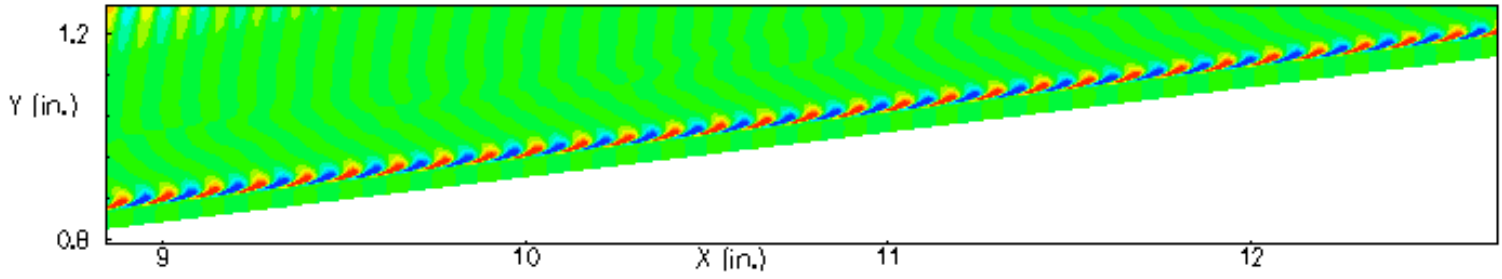

[d]

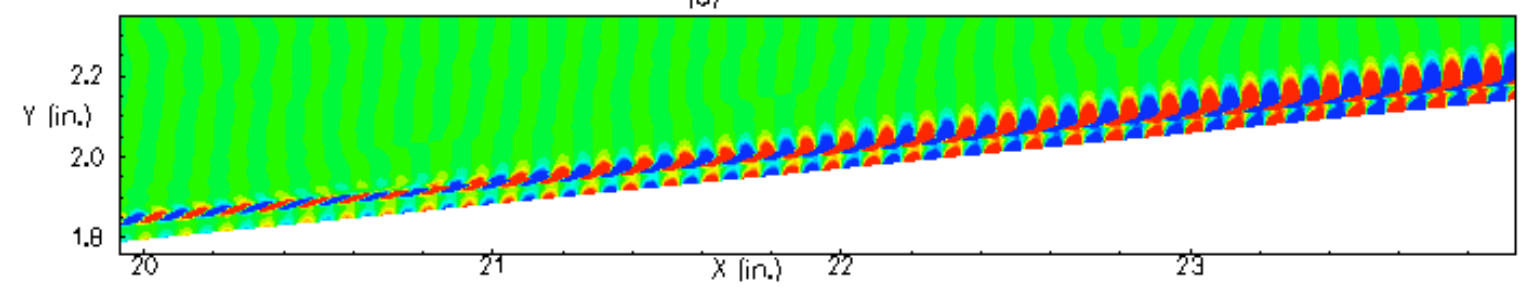

Figure 10. Expanded view of the contours of unsteady density fluctuations near the wall along the axial direction. 
(a)

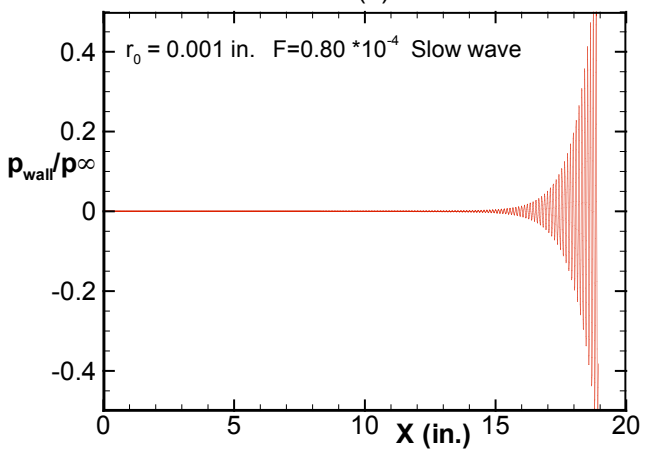

(c)

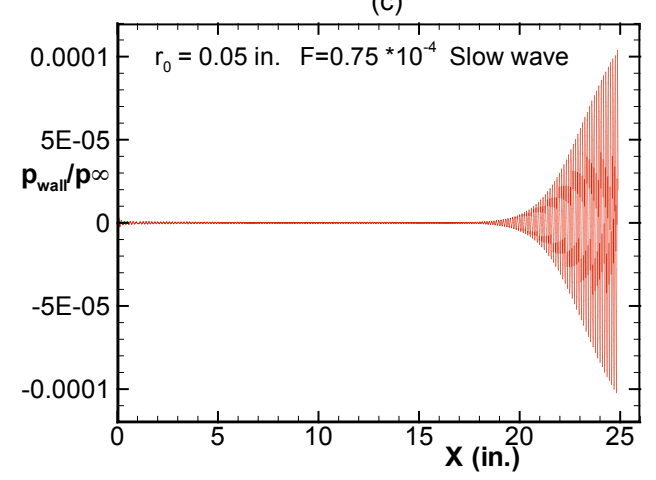

(e)

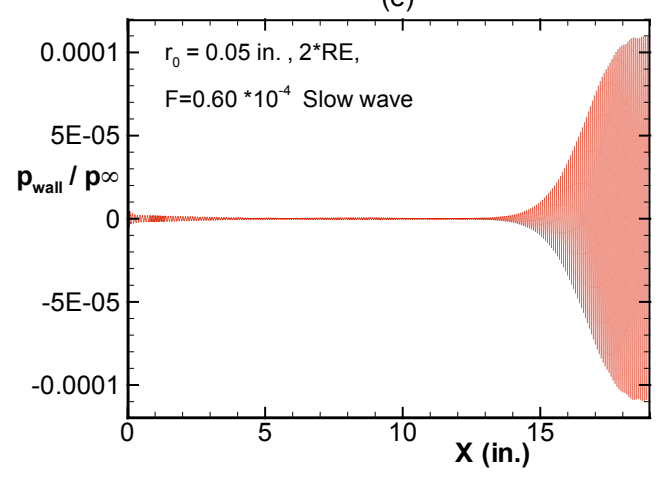

(g)

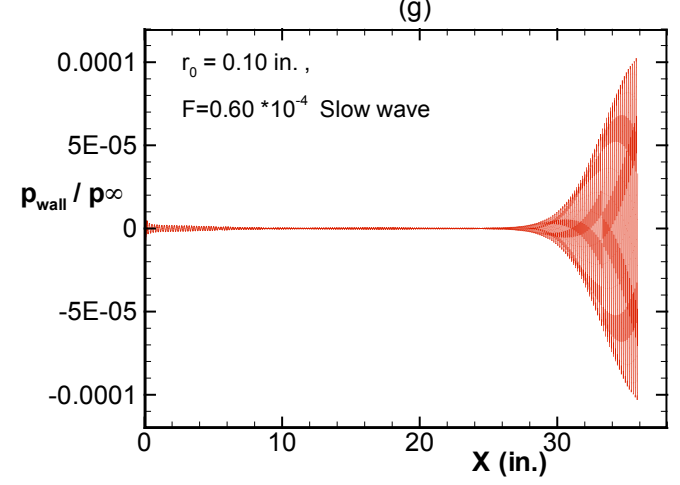

(b)

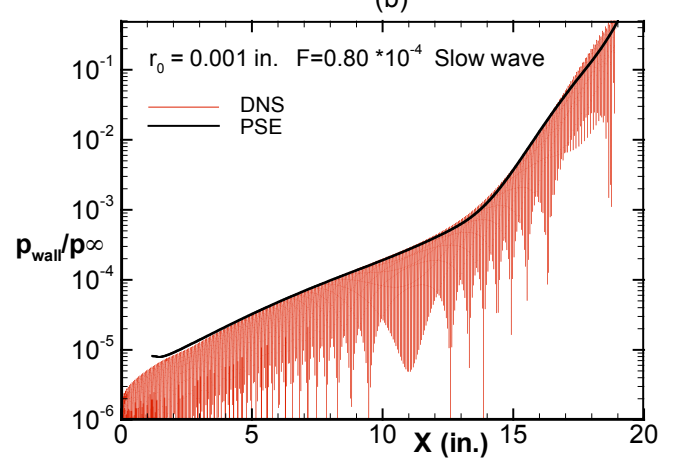

(d)
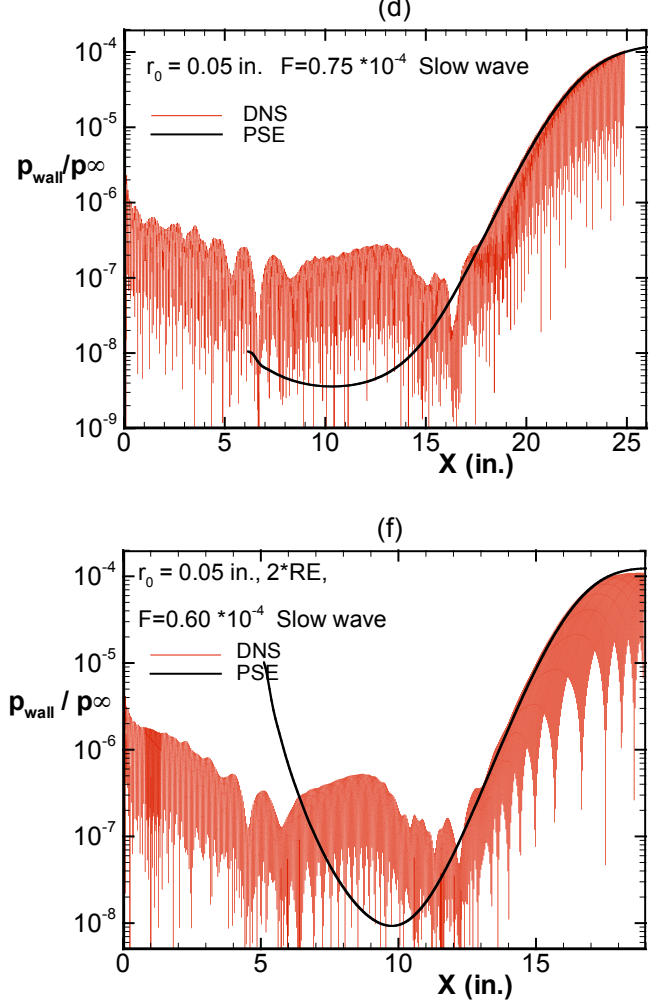

(h)

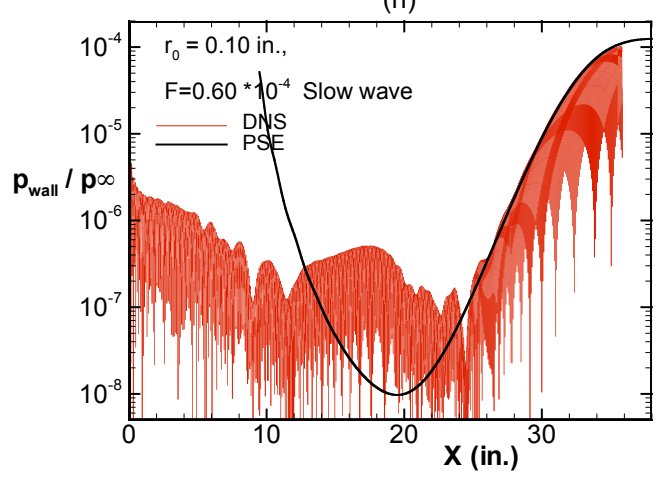

Figure 11. Amplitude of the pressure fluctuation on the wall (a), (c), (e), (g), and comparison with the PSE (b), (d), (f), (g). 

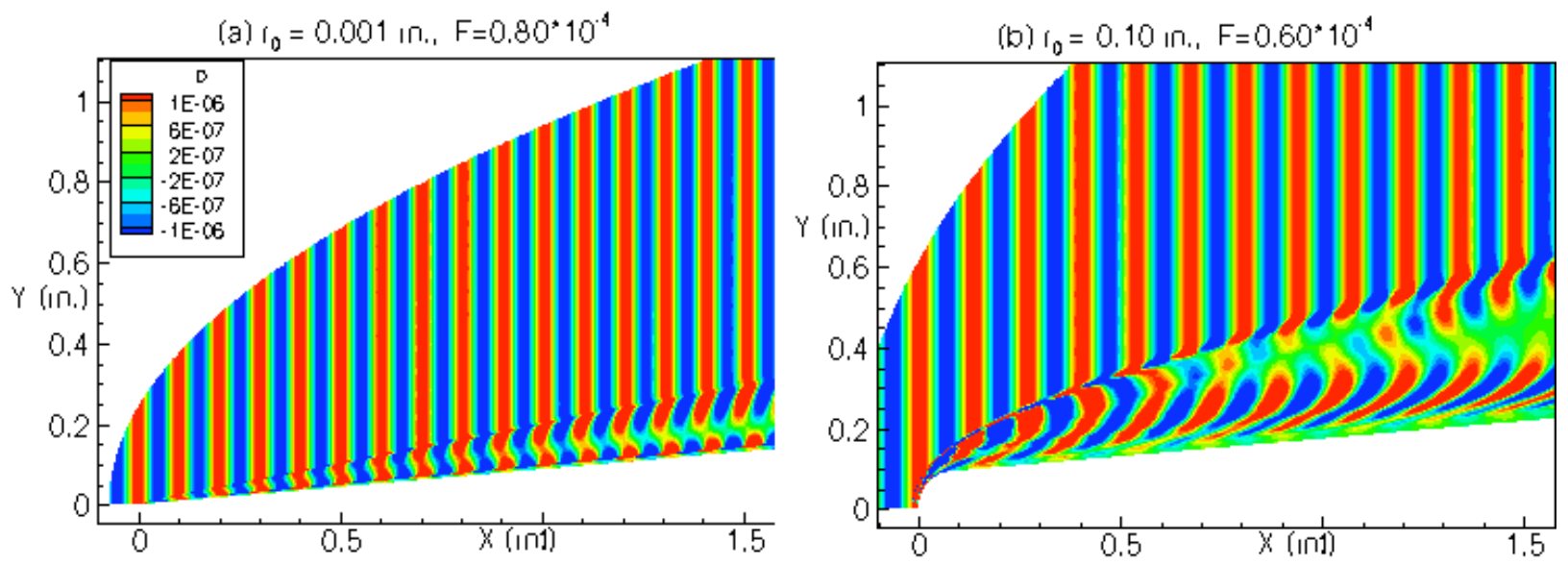

Figure 12. Contours of unsteady density fluctuations inside the boundary layer near the nose region for two bluntness cases (a) $r_{0}=0.001, F=0.80 \times 10^{-4}$ and (b) $r_{0}=0.10, F=0.60 \times 10^{-4}$. 